\title{
MicroRNA-143 targets CD44 to inhibit breast cancer progression and stem cell-like properties
}

\author{
ZHUANGQING YANG, DEDIAN CHEN, JIANYUN NIE, SHAOQIANG ZHOU, \\ JIANKUI WANG, QI TANG and XIAOJUAN YANG \\ Department of Mammary Glands, The Third Affiliated Hospital of Kunming Medical University, \\ Yunnan Tumor Hospital, Kunming, Yunnan 650118, P.R. China
}

Received November 21, 2014; Accepted September 16, 2015

DOI: $10.3892 / \mathrm{mmr} .2016 .5194$

\begin{abstract}
CD44 is closely linked to breast cancer progression; however, the regulatory functions of microRNAs (miRs) in breast cancer have yet to be fully elucidated. In order to investigate the regulation of CD44 by miRs in breast cancer, the present study isolated CD44+ and CD44- breast cancer cells by flow cytometry, revealing that CD44+ cells were enriched in transplanted compared with those in primary breast cancers, and that their proliferation and stem-cell sphere formation ability were enhanced. A miRNA array assay indicated that miR-143 expression in CD44+ breast cancer cells was lower than that in CD44- cells. Furthermore, miR-143 was decreased in breast cancer tissues and cell lines compared with that in normal tissues and cells. Restoration of miR-143 expression in CD44+ breast cancer cells inhibited their proliferation and sphere formation. A luciferase reporter assay demonstrated that miR-143 directly tartgeted the 3'-untranslated region of CD44. In addition, miR-143 inhibited metastasis-associated features in breast cancer and reduced tumor growth in a mouse model of breast cancer. In conclusion, the results of the present study demonstrated that miR-143 inhibited the progression and stem-cell properties of breast cancer cells by targeting CD44.
\end{abstract}

\section{Introduction}

Breast cancer is the most common type of cancer in women. It is known that cancer stem cells have critical roles in breast cancer progression. CD44 is considered to be a stem cell marker for breast cancer (1) and has important roles in processes of cancer progression, including proliferation, cell cycle, stem cell properties and metastasis $(2,3)$. The cancer

Correspondence to: Dr Jianyun Nie, Department of Mammary Glands, The Third Affiliated Hospital of Kunming Medical University, Yunnan Tumor Hospital, 519 Kunzhou Road, Kunming, Yunnan 650118, P.R. China

E-mail: jianyun-nie@sohu.com

Key words: microRNA-143, CD44, breast cancer, stem cell, tumorigenesis stem cell (CSC) sub-population is, at least in part, responsible for therapy resistance and metastasis of cancer cells (4). Studies have shown that CSCs and cells undergoing epithelial-mesenchymal transition (EMT), which have CSC-like properties, have crucial roles in drug resistance and early cancer metastasis of numerous cancer types, including breast cancer (5-8). In spite of the abundance of studies on CSCs, the molecular mechanisms associated with their role in tumor progression and resistance require further study.

Emerging evidence indicated the critical roles of microRNAs (miRNAs/miRs) in cancer (9). miRNAs are small and non-coding nucleotides which regulate gene expression by imperfectly binding to the 3'-untranslated regions (3'UTR) of target mRNAs, leading to the inhibition of their translation (10). miRNAs are known as important factors regulating organ development, cell proliferation, differentiation, apoptosis and cell cycle $(9,10)$. In the recent years, an increasing number of miRNAs have been identified to be aberrantly upor downregulated in various types of cancer, and to be either positively or negatively associated with their development and progression $(9,10)$.

A previous microarray study performed by our group showed that several stem cell markers, such as CD44, were upregulated in transplanted breast cancers (unpublished data). As CD44 was increased to a greater extent than the other stem cell markers, the present study isolated CD44+ and CD44+ cells to assess differences in their tumorigenicity. A miRNA array performed in CD44+ breast cancer cells demonstrated that miR-143 was markedly downregulated. Therefore, the present study assessed the effects of miR-143 targeting CD44 on cellular properties associated with metastasis and cancer progression. The present study suggested that miR-143 functions as a tumor suppressor in breast cancer.

\section{Materials and methods}

Cell culture. The breast cancer cell lines BT474, MDA231, MDA468, MCF7, SKBR3, and SUM102 were obtained from the American Type Culture Collection (ATCC, Manassas, VA, USA). The cells were cultured in Dulbecco's modified Eagle's medium (DMEM; Gibco-BRL; Thermo Fisher Scientific, Waltham, MA, USA) with $10 \%$ fetal bovine serum, penicillin $(100 \mathrm{U} / \mathrm{ml})$ and streptomycin $(100 \mu \mathrm{g} / \mathrm{ml})$ (all from 
Sigma-Aldrich, St. Louis, MO, USA). Cells were cultured in a humidified atmosphere containing $5 \% \mathrm{CO}_{2}$ at $37^{\circ} \mathrm{C}$. The MCF10A normal human breast cell line was purchased from ATCC. MCF10A cells were cultured in DMEM/F-12 containing 5\% horse serum (Thermo Fisher Scientific), $100 \mathrm{U} / \mathrm{ml}$ penicillin, $100 \mu \mathrm{g} / \mathrm{ml}$ streptomycin, $10 \mu \mathrm{g} / \mathrm{ml}$ insulin (Sigma-Aldrich) and $20 \mathrm{ng} / \mathrm{ml}$ epidermal growth factor (R\&D Systems, Minneapolis, MN, USA).

Clinical specimens. Primary breast cancer tissue samples and adjacent normal tissue samples were obtained at the Third Affiliated Hospital of Kunming Medical University (Kunming, Yunnan) from 2006 to 2011. None of the patients (mean age, 51.3 years) received chemotherapy or radiotherapy prior to surgery. Hematoxylin and eosin staining was used to confirm the diagnosis of breast cancer (11-13). Tissue collection protocols were approved by the Ethics Committee of the First Affiliated Hospital of Soochow University (Suzhou, China).

miRNA precursor expression profiling. Total RNA was isolated from the CD44+ and CD44- breast cancer cells using TRIzol (Invitrogen; Thermo Fisher Scientific). The RNA concentration was quantified using the ND-1000 micro-spectrophotometer (NanoDrop; Thermo Fisher Scientific). The integrity of the RNA was evaluated using the Agilent 2100 Bioanalyzer (Agilent Technologies, Palo Alto, CA, USA). Total RNA was used for reverse-transcription (RT) reaction to produce cDNA using gene-specific primers to 222 miRNA precursors plus $18 \mathrm{~S}$ ribonucleic (r)RNA. Real-time quantitative polymerase chain reaction (qPCR) was used to examine the expression of the 222 miRNA precursors. All primers and all components of the PCR mixtures were provided by Shanghai Kangcheng (Shanghai, China) and reactions were performed in an ABI Prism ${ }^{\circledR} 7900$ HT real-time PCR apparatus (Thermo Fisher Scientific). The thermocycling conditions were as follows: 1 cycle at $50^{\circ} \mathrm{C}$ for $2 \mathrm{~min}, 1$ cycle at $95^{\circ} \mathrm{C} 10 \mathrm{~min}$ and $95^{\circ} \mathrm{C}$ for $15 \mathrm{sec}$ and $60^{\circ} \mathrm{C}$ for $30 \mathrm{sec}$ ( 40 cycles). The mean cycle threshold (CT) value was determined from duplicate PCRs. The amount of miRNAs, was normalized to $18 \mathrm{~S}$ rRNA and the relative

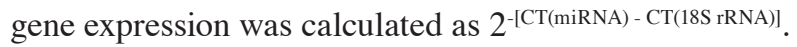

Plasmid construction. To construct the miR-143 expression vector (P-miR-143), a fragment including the mature miR-143 sequence was amplified from MRC-5-cell genomic DNA (Biomat, Bejing, China) and then cloned into the BamHI and $\mathrm{XbaI}$ sites in pcDNA3.1 (Invitrogen). The following primers were used: Forward, 5'-CGGCTAGCCGTTCAGTTTCTA AACCAGCAC-3' and reverse, 5'-GCTCTAGAGCCTACA TGGCATCATCCTTC-3' (Invitrogen). CD44 was predicted as the target gene of miR-143 using TargetScan (http://www. targetscan.org/vert_61/). The 3'UTR of CD44 was amplified from MRC-5 cells by PCR (11). The primers for CD44 were as follows: Forward, 5'-GTTCCTGGACTGATTTC-3' and reverse, 5'-TTACTCTGCTGCGTTG-3' (Invitrogen). The thermocycling conditions were $94^{\circ} \mathrm{C}$ for 5 min followed by 25 cycles of $94^{\circ} \mathrm{C}$ for $30 \mathrm{sec}, 55^{\circ} \mathrm{C}$ for $30 \mathrm{sec}$ and $72^{\circ} \mathrm{C}$ for $1 \mathrm{~min}$, and a final extension at $72^{\circ} \mathrm{C}$, for $10 \mathrm{~min}$. These fragments were ligated into the NheI and $\mathrm{XbaI}$ sites with specific restriction enzymes (Promega Corp., Madison, WI, USA) based on the pmirGLO Dual-Luciferase miRNA Target Expression Vector (Promega Corp.), which included firefly luciferase and renilla luciferase. Firefly luciferase is a reporter monitoring mRNA regulation and renilla luciferase is a control reporter for normalization. The mutant 3'-UTR of CD44 was generated using the overlap-extension PCR method (RiboBio Co. Ltd., Guangzhou, China). All constructs were confirmed by DNA sequencing analysis (ABI3730 DNA sequencer; Thermo Fisher Scientific). For each group, $2 \times 10^{5}$ SKBR3 cells were transfected with miR-143 or CD44 using Lipofectamine 2000 (Invitrogen) according to the manufacturer's instructions. The miR-143 mimic, a nonspecific miR control, anti-miR143a and a nonspecific anti-miR control were all purchased from Sigma-Aldrich.

RNA isolation and RT-qPCR. The tissues were homogenized using TissueLyser II (Qiagen, Hilden, Germany) with natural extraction buffer and $10 \%$ sodium dodecyl sulfate (SDS).Total RNA from the tissues or cells transfected with miRNA-expressing plasmids was extracted using TRIzol (Invitrogen) according to the manufacturer's instructions. RT-qPCR was performed using Real-Time RT-PCR Master Mix containing SYBR Green I and hotstart Taq DNA polymerase (Toyobo, Osaka, Japan). CD44, CD133, c-myc and GAPDH primers were supplied by Sangon Biotech, Co., Ltd. (Shanghai, China) with the following sequences: miR-143 forward, 5'-TGAGATGAAGCACTGTAGCTC-3' and reverse, 5'-GCGAGCACAGAATTAATACGAC-3'; CD44 forward, 5'-GGACTCTGCCTCGTGCCG-3' and reverse, 5'-ATTGAA GCAATATGTGTCATAC-3'; CD133 forward, 5'-ACTCGG CTCCCTGTTGCTGCT-3' and reverse, 5'-GAGCCTGATATC CTGACCATTG-3'; OCT4 forward, 5'-GTCTTCTGCCTT TTAAAATCCA-3' and reverse, 5'-ACTCGGTTCTCGATA CTGGTT-3'; GAPDH forward, 5'-GGAAGGTGAAGGTCG GAGTCA-3' and reverse, 5'-GGAAGATGGTGATGGGAT TTC-3'; and U6 small nuclear (sn)RNA forward, 5'-CTCGCT TCGGCAGCACA-3' and reverse, 5'-AACGCTTCACGAATT TGCCT-3'. PCR was performed in an ABI Prism ${ }^{\circledR} 7900 \mathrm{HT}$ real-time PCR apparatus with the following conditions: $95^{\circ} \mathrm{C}$ for $1 \mathrm{~min}$; followed by 40 cycles of $95^{\circ} \mathrm{C}$ for $10 \mathrm{sec}$ and $65^{\circ} \mathrm{C}$ for $40 \mathrm{sec}$. Relative gene expression was determined using the $\Delta \triangle \mathrm{CT}$ method with normalization to the housekeeping gene GAPDH or U6 snRNA using the formula $2^{-\Delta \Delta C T}$.

Fluorescence-activated cell sorting (FACS). Cells $\left(10^{4}\right)$ were suspended in FACS buffer [phosphate-buffered saline (PBS) containing $0.1 \%$ bovine serum albumin and $0.1 \%$ Triton X100] and incubated in fluorescein isothiocyanate-conjugated anti-CD44 antibody for $15 \mathrm{~min}$ at room temperature. Cells were then washed with PBS and re-suspended in PBS for FACS analysis (6HT; Guava ${ }^{\circledR}$; Merck-Millipore, Billerica, MA, USA) and to isolate CD44+ and CD44- negative cells.

MTT assay. Breast cancer cells were transfected with miRNA-containing plasmids for $24 \mathrm{~h}$, collected and re-seeded in a 96-well plate at $5 \times 10^{3}$ cells per well. The control group was transfected with control miRNA. The breast cancer cells were incubated at $37^{\circ} \mathrm{C}$ for $0,1,2,3,4$ or 5 days and $10 \mu 1$ MTT ( $5 \mathrm{mg} / \mathrm{ml}$; Sigma-Aldrich) was added, followed by further culture for $4 \mathrm{~h}$. The medium was discarded and $150 \mu \mathrm{l}$ 
dimethyl sulfoxide (Sangon, Shanghai, China) was added to each well to dissolve the formazan crystals completely. The absorbance at $570 \mathrm{~nm}$ was measured using an ELISA reader (SM800; Utrao Medical Instruments, Shanghai, China).

Colony formation assay. Breast cancer cells were transfected with miRNA-containing plasmids for $24 \mathrm{~h}$ in six-well plates, digested with $0.1 \%$ trypsin containing $0.02 \%$ EDTA and re-suspended in medium at $10^{5}$ cells $/ \mathrm{ml}$. A total of 100 cells were added to each well of six-well plates and incubated at $37^{\circ} \mathrm{C}$ for $24 \mathrm{~h}$. At day 3, the medium was replaced. After 10 days, colonies which had formed (comprising at least 50 cells) were counted under a microscope (IX73; Olympus, Tokyo, Japan).

Tumor spheroid assay. Breast cancer cells were transfected with miRNAs or plasmids for $24 \mathrm{~h}$ in six-well plates. Single-cell suspensions were prepared and cells were plated on six-well ultralow attachment plates (Corning-Costar Inc., Corning, NY, USA) at a density of 1,000 cells $/ \mathrm{ml}$. Cells were grown in DMEM supplemented with 1\% N2 Supplement (Invitrogen), 2\% B27 Supplement (Invitrogen), $20 \mathrm{ng} / \mathrm{ml}$ human platelet growth factor (Sigma-Aldrich), $100 \mathrm{ng} / \mathrm{ml}$ epidermal growth factor (Invitrogen) and 1\% antibiotic-antimycotic (Invitrogen). After 10 days of culturing, the mammospheres were counted.

Western blot analysis. Total protein from the cells transfected with miRNAs or plasmids was extracted by lysis in radioimmunoprecipitation assay buffer containing $1 \mathrm{X}$ protease inhibitor cocktail (both from Santa Cruz Biotechnology, Inc., Dallas, TX, USA) and quantified using the Bradford assay (Bio-Rad Laboratories, Inc., Hercules, CA, USA). Equal amounts of protein $(50 \mu \mathrm{g} /$ lane $)$ were separated by $12.5 \%$ SDS-PAGE and then transferred onto polyvinylidene difluoride (PVDF) membranes (Millipore, Billerica, MA, USA) at $55 \mathrm{~V}$ for $4 \mathrm{~h}$ at $4^{\circ} \mathrm{C}$. The protein in the PVDF membranes was blocked in 5\% fat-free dry milk in Tris-buffered saline (TBS). The PVDF membranes were incubated with primary antibodies in TBS containing Tween 20 (TBST; Sangon) overnight at $4^{\circ} \mathrm{C}$ and washed three times with TBST, followed by incubation with horseradish peroxidase (HRP)-conjugated secondary antibodies in TBS for $1 \mathrm{~h}$ at room temperature. The following antibodies were used: CD44 (156-3C11) mouse monoclonal antibody (cat. no. 3570; dilution, 1:1,000; Cell Signaling Technology, Danvers, MA, USA), fibronectin antibody (A-11; (cat. no. sc-271098; dilution, 1:500), E-cadherin antibody (67A4; cat. no. sc-21791; dilution, 1:1,000) and vimentin antibody (J144; cat. no. sc-53464; dilution, 1:1,000) (all from Santa Cruz Biotechnology, Inc) as well as anti- $\alpha$ SMA (1A4; dilution, 1:2,000; Sigma-Aldrich). As secondary antibodies, HRP-conjugated goat anti-mouse immunoglobulin G (IgG) (cat no. sc-2005; dilution, 1:3,000) and HRP-conjugated goat anti-rabbit IgG (cat no. sc-2004; dilution, 1:2,000) were used (both from Santa Cruz Biotechnology, Inc.). Protein bands were visualized using an enhanced chemiluminescence Western Blotting kit (Pierce Biotechnology, Inc., Rockford, IL, USA). Protein bands were captured using a Canon CanoScan LiDE 220 image scanner (Canon, Tokyo, Japan).

Transwell invasion assay. Breast cancer cells were transfected with miRNA-containing plasmids in six-well plates for $24 \mathrm{~h}$ and then collected for a migration assay. A total of $1 \times 10^{5}$ breast cancer cells were seeded in the upper chambers of a Transwell system with Matrigel-coated Membranes (24-well insert; pore size, $8 \mu \mathrm{m}$; Corning-Costar). The cells invaded through the Matrigel-coated membrane were fixed with methanol, stained with $0.1 \%$ crystal violet (Sigma-Aldrich) and counted under a light microscope (magnification, 40x; three random fields per well).

Mouse model of breast cancer. Six-week old female BALB/c nude mice $(n=15)$ were purchased from the Shanghai Laboratory Animal Center (Shanghai, China). The animals were maintained under regulated pathogen-free conditions, and kept with a $12-\mathrm{h}$ light/dark cycle at $25^{\circ} \mathrm{C}$ and $40-60 \%$ relative humidity. Mice were treated with miRNAs or the control via injection into the right flank. Tumor size was calculated using a vernier caliper. The experiments were approved by the Ethics Committee of Yunnan Tumor Hospital (Kunming, China). To establish the mouse model of breast cancer, $2 \times 10^{5}$ SKBR3 cells transfected with miR-143-overexpressing vector were inoculated subcutaneously into the dorsal flanks of the mice (five per group). The tumor size was measured every seven days. At 28 days following inoculation the mice were sacrificed, and tumors were harvested, weighed and measured. The tumor volumes were calculated using the following formula: Largest diameter $\mathrm{x}$ (diameter perpendicular to the largest diameter $)^{2} / 2$. All procedures involved were performed following institutional guidelines.

Statistical analysis. Values are expressed as the mean \pm standard deviation. Associations between protein expression levels in the breast tissue microarray were assessed using Spearman's rank correlation test. Comparisons between different groups were performed by one- or two-way analysis of variance, followed by Bonferoni's multiple comparisons tests using GraphPad Prism statistical analysis software (GraphPad Software, Inc., La Jolla, CA, USA). P $<0.05$ was considered to indicate a statistically significant difference between values.

\section{Results}

CD44 promotes progression of breast cancer. Microarray data from a previous study performed by our group (unpublished data) showed that expression levels of CD44, CD133 and OCT4 were significantly increased in transplanted cells compared to those in primary breast cancer tissues. In the present study, overexpression of CD44 was verified using real-time RT-qPCR (Fig 1A). CD44+ cells were quantified and isolated by flow cytometry (Fig. 1B), revealing that CD44+ cells were present at a larger proportion in transplanted tumor cells than in primary tumor cells. Furthermore, the biological properties of CD44+ cells were analyzed. The proliferation of CD44+ breast cancer cells was increased compared with that of CD44- cells (Fig. 1C and D). In addition, the sphere formation of CD44+ cells was increased compared with that of CD44- cells (Fig. 1E). These results indicated that CD44+ breast cancer cells had stem-like properties.

miR-143 is downregulated in CD44+ breast cancer cells. miRNA profiling was performed in CD44+ and CD44- breast 

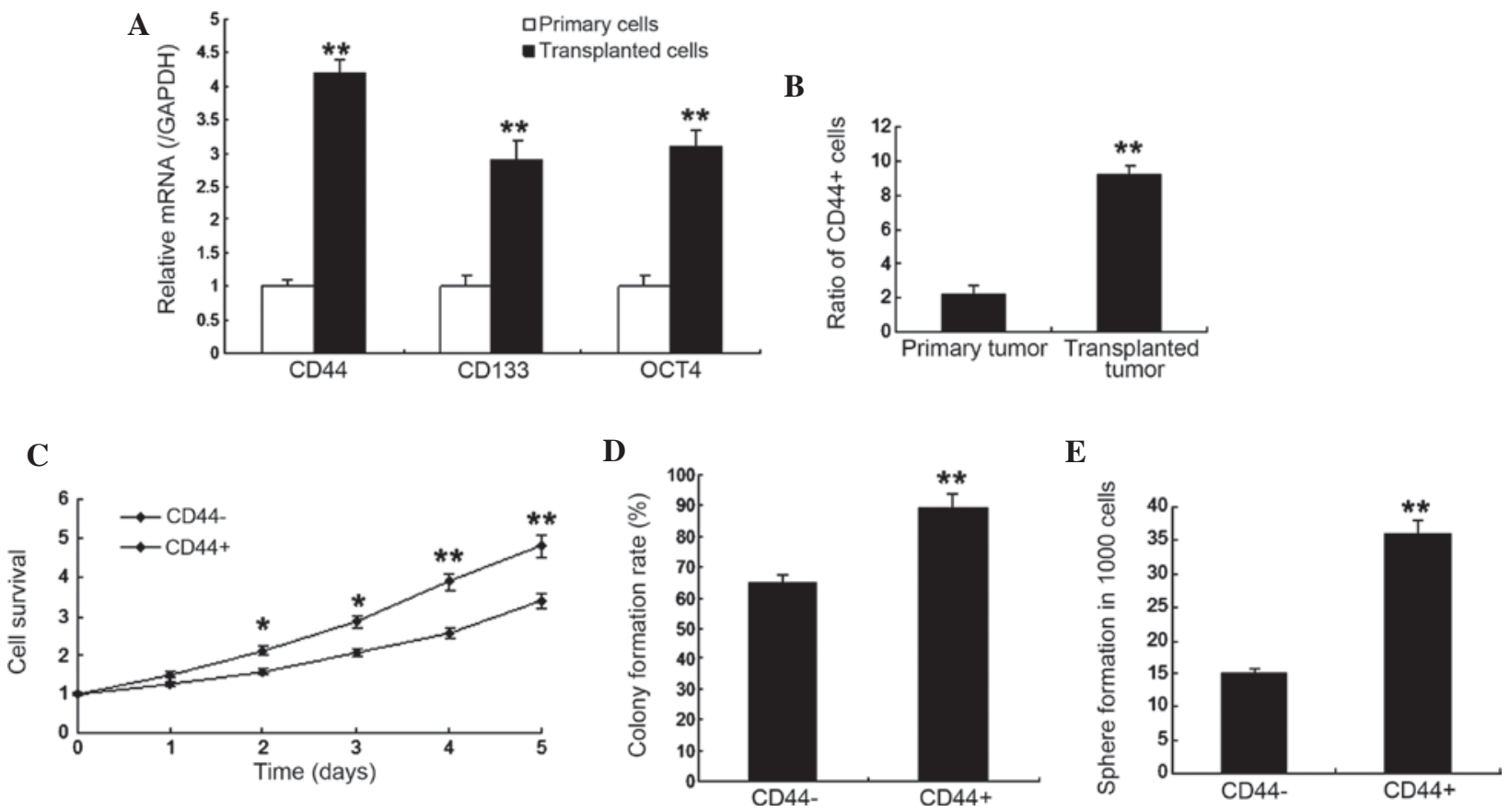

Figure 1. CD44 promotes proliferation and stem-cell properties of breast cancer cells. (A) CD44, CD133 and C-myc expression in primary tumor cells and transplanted cells from xenograft tumors were verified by reverse-transcription quantitative polymerase chain reaction analysis. (B) Ratio of CD44+ cells from primary or transplanted tumors as determined by flow cytometry. (C) Proliferation of CD44+ and CD44- cells was analyzed using an MTT assay following $0,1,2,3,4$ or 5 days of culturing. ${ }^{*} \mathrm{P}<0.05$; ${ }^{* * *} \mathrm{P}<0.01$ vs CD44- cells. (D) The colony formation ability of CD44+ and CD44- cells was assessed using a 10 -day clonogenic assay. (E) The stem-cell sphere formation was assessed in CD44- and CD44- cells. Values are expressed as the mean \pm standard deviation. ${ }^{* *} \mathrm{P}<0.01$ vs primary tumor or CD44- cells, respectively.

A

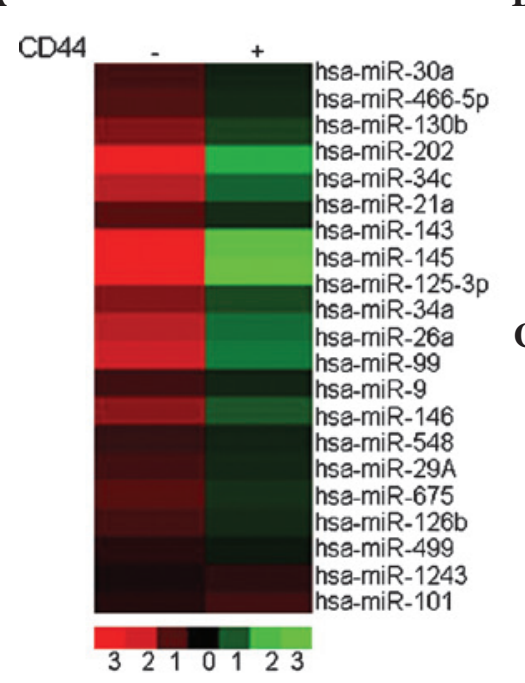

B

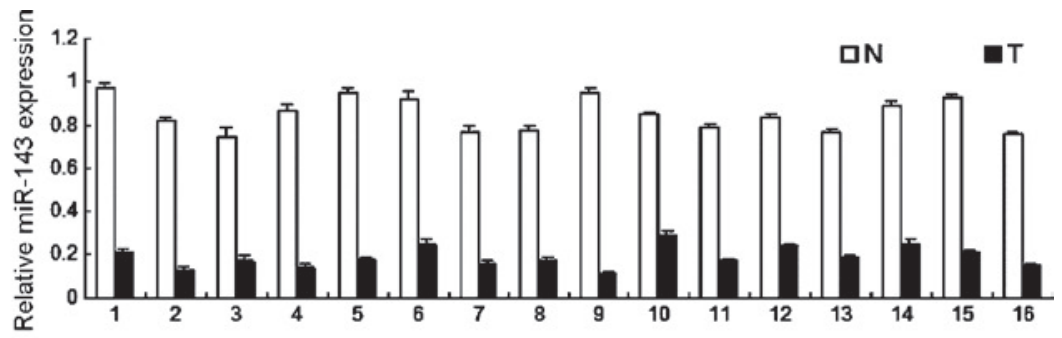

C

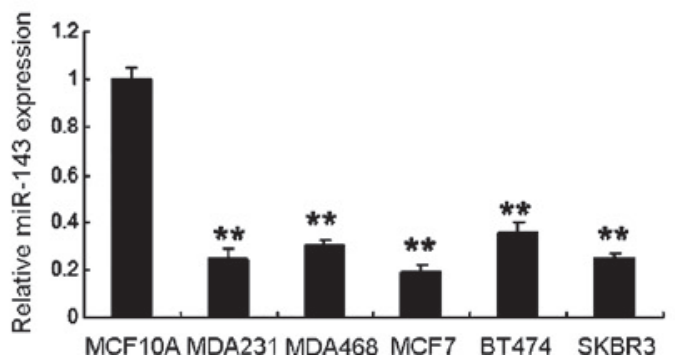

Figure 2. Loss of miR-143 in CD44+ breast cancer cells. (A) CD44+ and CD44- cells from xenograft transplants were subjected to a miR array assay. (B) RT-qPCR analysis revealed that miR-143 expression levels in human breast cancer specimens $(\mathrm{n}=16)$ were significantly lower than those in normal tissues $(\mathrm{n}=16)$. U6 RNA was used as the internal control. (C) RT-qPCR analysis of miR-143 in breast cancer cells lines and a normal breast cell line (MCF10A). Values are expressed as the mean \pm standard deviation of at least three experiments. ${ }^{* *} \mathrm{P}<0.01$ vs MCF10A. miR, micro RNA; N, non-tumorous tissue; $\mathrm{T}$, tumorous tissue; hsa, Homo sapiens, RT-qPCR, reverse-transcription quantitative polymerase chain reaction; miR, microRNA.

cancer cells, revealing that miR-143 was significantly downregulated in $\mathrm{CD} 44+$ breast cancer cells compared to that in CD44- cells (Fig. 2A). Next, the expression of miR-143 in 16 human breast cancer samples and 16 normal tissues was examined by real time RT-qPCR. As shown in Fig. 2B, miR-143 expression levels in tumor samples were lower than those in normal tissues. In analogy with this result, miR-143 expression was lower in human breast cancer cell lines compared with that in a normal breast cell line (Fig. 2C). These results indicated that miR-143 may suppress breast cancer progression.

CD44 is a novel target gene of miR-143 in breast cancer cells. To investigate the association of CD44 and miR-143, a Targetscan analysis (http://www.targetscan.org/) was 


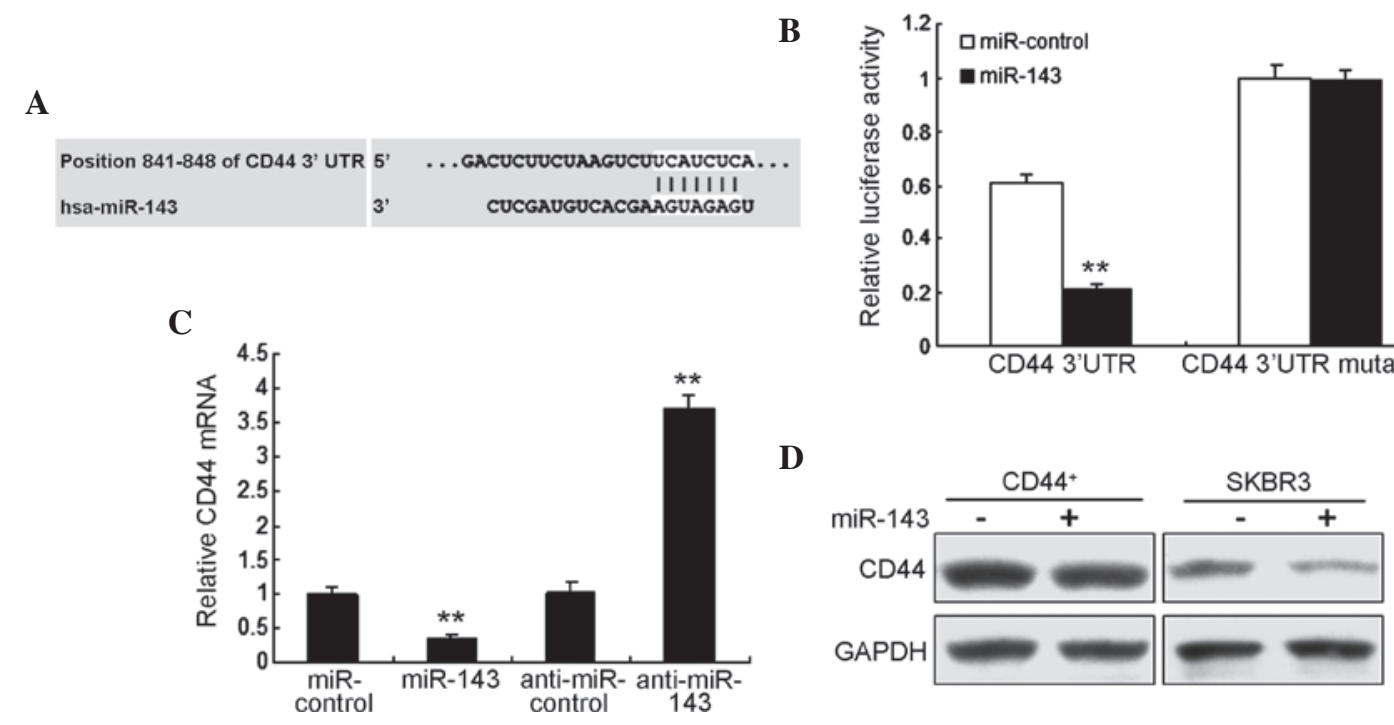

Figure 3. CD44 is a novel target gene of miR-143 in breast cancer cells. (A) The 3'-UTR of the CD44 gene contains binding sites for miR-143 according to bioinformatics analysis. (B) miR-143 suppressed the expression of a luciferase reporter gene harbouring the 3'-UTR of CD44. SKBR3 cells were transfected with luciferase vector containing the wild-type or mutated 3'-UTR of the CD44 gene with simultaneous transfection with miR-143 or control miR. Luciferase activity was assessed following incubation for $48 \mathrm{~h}$ later. (C) Restoration of miR-143 decreased the expression of CD44 mRNA in CD44+ breast cancer cells. SKBR 3 cells were transfected with miR-143 or control miRNA for $48 \mathrm{~h}$ and subjected to reverse-transcription quantitative polymerase chain reaction analysis. (D) Restoration of miR-143 decreased the protein expression of CD44 in CD44- breast cancer cells. SKBR3 cells were transfected with miR-143 or control miRNA for $48 \mathrm{~h}$ and subjected to western blot analysis. GAPDH was used as a loading control. Values are expressed as the mean \pm standard deviation of at least three independent experiments. ${ }^{* *} \mathrm{P}<0.01$ vs miR-control group. miR, microRNA; UTR, untranslated region; hsa, Homo sapiens.

A

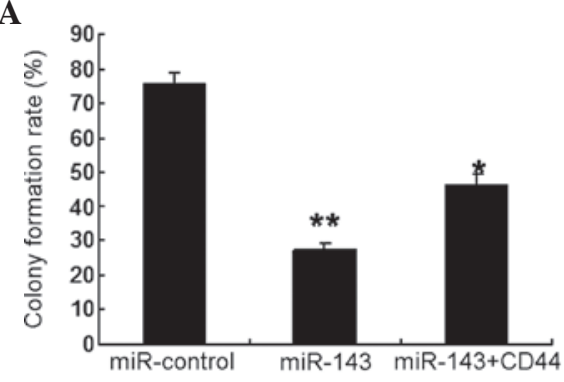

C

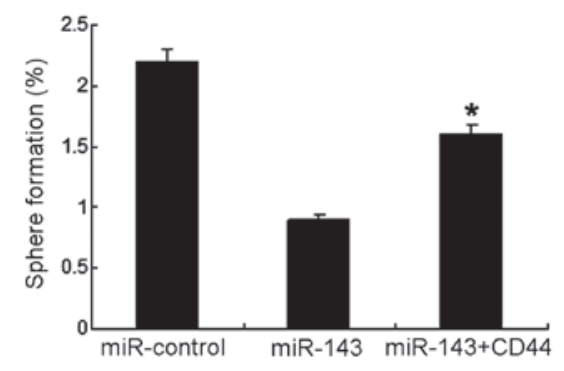

B

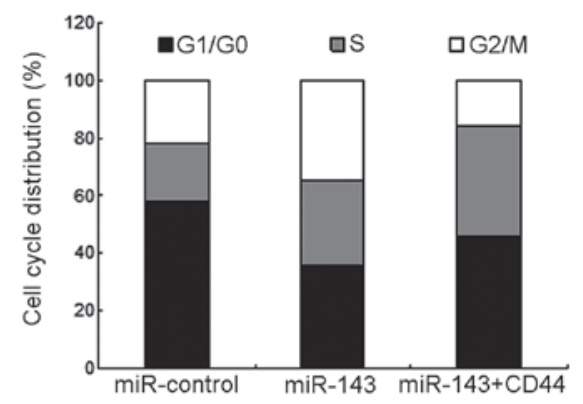

D

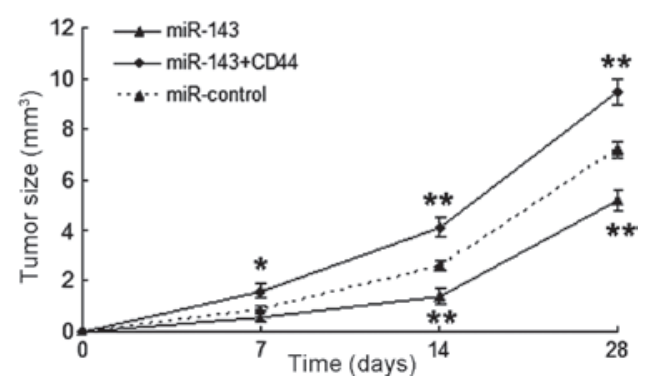

Figure 4. miR-143 inhibits the proliferation and stem cell properties of breast cancer cells by targeting CD44. SKBR3 cells were transfected with control vector or miR-143 overexpression vector with or without CD44 overexpression vector. (A) To assess colony formation ability, SKBR3 cells were subjected to a 10-day clonogenic assay. (B) The cell-cycle distribution of SKBR3 cells was assessed by flow cytometry. (C) Stem-cell sphere formation of SKBR3 cells was assessed. (D) The tumor formation ability of CD44+ and CD44- cells with or without miR-143 expression was assessed in a nude mouse model. Values are expressed as the mean \pm standard deviation of at least three experiments. ${ }^{*} \mathrm{P}<0.05 ;{ }^{* * *} \mathrm{P}<0.01$ vs miR-control group. miR, microRNA.

performed, which indicated that CD44 may be directly suppressed by miR-143 (Fig. 3A). To experimentally verify this interaction, a luciferase reporter assay was performed (Fig. 3B), which demonstrated that following transfection with miR-143, the luciferase activity of a reporter plasmid containing the wild-type 3'UTR of CD44 in CD44+ cells was lower than that in control cells, while luciferase activity in cells transfected with a reporter plasmid containing a mutated 3'UTR of CD44 was markedly elevated and not affected by miR-143. Furthermore, RT-qPCR analysis demonstrated that compared with the control, endogenous CD44 mRNA levels were downregulated in the cells with miR-143 overexpression (Fig. 3C). In addition, western blot analysis showed that following transfection of the CD44+ SKBR3 breast cancer 
A

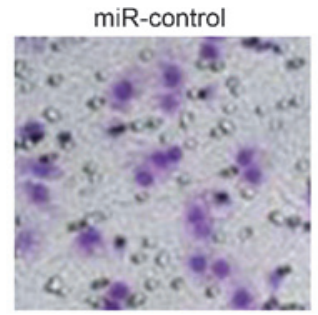

B

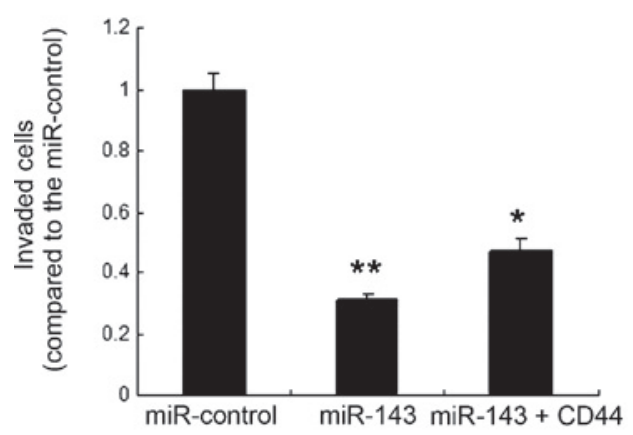

$\operatorname{miR}-143$

C
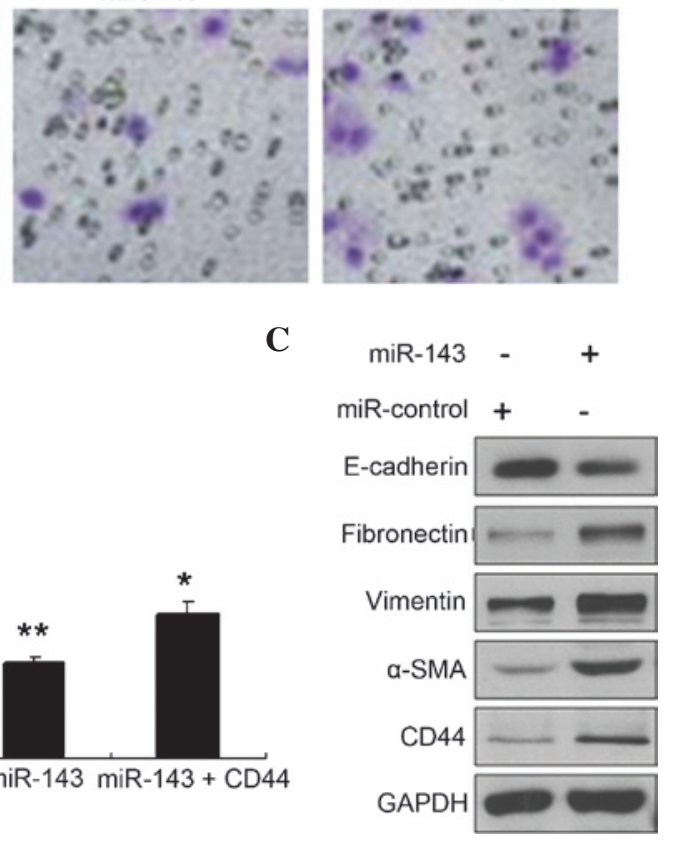

Figure 5. miR-143 inhibits metastasis-associated properties of breast cancer cells. (A) A Transwell assay of SKBR3 cells transfected with control miR or miR-143 with or without CD44 indicated that miR-143 inhibited breast cancer-cell invasion. Representative images of crystal violet-stained cells on the lower side of the membrane, which had transgressed through the Matrigel (magnification, x40). (B) Quantification of invaded cells in A. Values are expressed as the mean \pm standard deviation. "P $<0.05 ;{ }^{*} \mathrm{P}<0.01$ vs miR-control group. (C) Western blot analysis of breast cancer cells transfected with miR-143 or control-miR for $48 \mathrm{~h}$ showed that miR-143 altered the expression of epithelial-mesenchymal transition-associated markers E-cadherin, fibronectin, vimentin, $\alpha$-SMA and CD44. GAPDH was used as a loading control. All experiments were performed at least three times. miR, microRNA; SMA, smooth muscle actin.

cells with miR-143, CD44 was decreased (Fig 3D), revealing that miR-143 regulated endogenous CD44 expression in breast cancer cells. These results clearly indicated that CD44 is a novel target gene of miR-143.

miR-143 inhibits progression and stem cell-like properties of breast cancer cells by targeting CD44. To explore the roles of miR-143 in cell survival and proliferation, SKBR3 cells were infected with miR-143 plasmid or control plasmid. A colony formation assay demonstrated that miR-143 inhibited the colony formation rate of SKBR3 cells (Fig. 4A). Furthermore, cell cycle analysis indicated that SKBR3 cells with miR-143 expression showed a decrease in the G1-phase population and an increase in S-phase population (Fig. 4B). Next, the self-renewal potential of breast cancer cells was examined. The stem-cell sphere formation assay revealed that miR-143 overexpressing CD44+ cells showed a significantly decreased ability of self renewal, with the sphere-formation rate being reduced by $87 \%$ (Fig. 4C). Furthermore, the effects of miR-143 on breast cancer cells were assessed in vivo using a mouse model. The tumor growth curve indicated that miR-143 reduced breast cancer formation (Fig. 4D).

miR-143 inhibits breast cancer cell metastasis by targeting $C D 44$. To further elucidate the role of miR-143 in breast cancer metastasis and EMT, CD44+ cells were infected with miR-143-containing vector and subjected to a Transwell assay, which indicated that upregulation of miR-143 significantly decreased the invasive capacity of breast cancer cells (Fig. 5A and B). In addition, the expression of EMT markers was assessed by western blot analysis. The results showed that mesenchymal markers fibronectin, vimentin and $\alpha$-SMA were decreased, while epithelial marker E-cadherin was increased in breast cancer cells with miR-143 overexpression (Fig. 5C). These results indicated that miR-143 suppressed metastasis and EMT of breast cancer cells.

\section{Discussion}

Cell surface markers of CSCs can help distinguish, isolate and purify these tumor-initiating cells for further biological investigation. A previous study by our group showed a upregulation of stem-cell markers in CSCs, including CD44 (unpublished data). The present study investigated cell surface protein CD44, which is commonly used as a stem cell marker to isolate breast cancer stem cells (1). A mammosphere formation assay was used to show that the self-renewal ability of CD44+ breast cancer cells in serum-free medium was higher than that in CD44- cells. Furthermore, an MTT and a clonogenic assay revealed that CD44+ breast cancer cells possessed a greater viability and a higher colony formation rate than CD44- cells. These experiments demonstrated that CD44+ breast cancer cells exhibited higher tumorigenicity and self-renewal ability than those of CD44- cells, and that they had cancer stem-like properties.

Bioinformatics analysis revealed that CD44 was a potential target gene of miR-143, which was experimentally verified using a luciferase activity assay. Furthermore, it was demonstrated that miR-143 inhibited breast cancer-cell proliferation as well as their stem cell-like properties and EMT by down-regulating CD44. The EMT is a key developmental process, which is frequently activated during cancer-cell invasion and metastasis, 
and which may promote resistance to chemotherapy (5). Loss of epithelial cell markers such as E-cadherin is considered to be a major hallmark of the EMT (5-8). E-cadherin is a classical member of the cadherin superfamily, which maintains epithelial cell plasticity. Mesenchymal cell markers usually induce EMT and metastasis of cancer. Accumulating evidence suggested that the EMT is closely associated with the acquisition of stem cell-like properties of cancer cells (6). The present study was the first to indicate that miR-143 suppresses EMT of CD44+ breast cancer cells and breast cancer tumorigenesis.

Previous studies indicated that low expression of miR-143 is closely linked to cancer development and identified it as a tumor suppressor (11-14). miR-143 regulates various target genes, including Limk1 (15), SDC1 (16), COX-2 (17), PKCe (18), MACC1 (19) and KRAS (20), in non-small cell lung cancer, melanoma, gastric cancer, breast cancer and prostate cancer. The present study showed that miR-143 inhibited breast cancer via CD44 expression.

Previous studies have indicated that miR-143 could suppress cell proliferation, glycolysis, metastasis and induce apoptosis in cancer cells (15-20). The present study demonstrated that miR-143 inhibited breast cancer cell proliferation assayed by MTT, colony formation and mammosphere formation by targeting CD44. The in vivo study also demonstrated that miR-143 suppressed breast cancer growth. The results also demonstrated that miR-143 inhibited breast cancer cell metastasis, including invasion and EMT.

In conclusion, the present study indicated that miR-143 functions as a tumor suppressor in breast cancer and that low miR-143 expression in breast cancer tissues may be an unfavorable prognostic factor. The association between miR-143 and CD44 in breast cancer tissues requires further study. To the best of our knowledge, the present study was the first to demonstrate that downregulation of miR-143 increased CD44 expression in breast cancer cells, which enhances their CSC-like properties; these are linked with breast cancer progression, metastasis and resistance to chemotherapy.

\section{References}

1. Kasai T, Chen L, Mizutani A, Kudoh T, Murakami H, Fu L and Seno M: Cancer stem cells converted from pluripotent stem cells and the cancerous niche. J Stem Cells Regen Med 10: 2-7, 2014.

2. Visvader JE and Stingl J: Mammary stem cells and the differentiation hierarchy: Current status and perspectives. Genes Dev 28 : 1143-1158, 2014.
3. Geng SQ, Alexandrou AT and Li JJ: Breast cancer stem cells: Multiple capacities in tumor metastasis. Cancer Lett 349: 1-7, 2014.

4. Fu N, Lindeman GJ and Visvader JE: The mammary stem cell hierarchy. Curr Top Dev Biol 107: 133-160, 2014.

5. Steinestel K, Eder S, Schrader AJ and Steinestel J: Clinical significance of epithelial-mesenchymal transition. Clin Transl Med 3: 17, 2014.

6. Puisieux A, Brabletz T and Caramel J: Oncogenic roles of EMT-inducing transcription factors. Nat Cell Biol 16: 488-494, 2014.

7. Lamouille S, Xu J and Derynck R: Molecular mechanisms of epithelial-mesenchymal transition. Nat Rev Mol Cell Biol 15: 178-196, 2014.

8. Hao J, Zhang Y, Deng M, Ye R, Zhao S, Wang Y, Li J and Zhao Z: MicroRNA control of epithelial-mesenchymal transition in cancer stem cells. Int J Cancer 135: 1019-1027, 2014.

9. Vislovukh A, Vargas TR, Polesskaya A and Groisman I: Role of 3'-untranslated region translational control in cancer development, diagnostics and treatment. World J Biol Chem 5: 40-57, 2014.

10. van Rooij E and Kauppinen S: Development of microRNA therapeutics is coming of age. EMBO Mol Med 6: 851-864, 2014.

11. Yan X, Chen X, Liang H, Deng T, Chen W, Zhang S, Liu M, Gao X, Liu Y,Zhao C, et al: MiR-143 and miR-145 synergistically regulate ERBB3 to suppress cell proliferation and invasion in breast cancer. Mol Cancer 13: 220, 2014.

12. Ng EK, Li R, Shin VY, Siu JM, Ma ES and Kwong A: MicroRNA-143 is downregulated in breast cancer and regulates DNA methyltransferases 3A in breast cancer cells. Tumour Biol 35: 2591-2598, 2014.

13. Jiang S, Zhang LF, Zhang HW, Hu S, Lu MH, Liang S, Li B, Li Y, Li D, Wang ED and Liu MF: A novel miR-155/miR-143 cascade controls glycolysis by regulating hexokinase 2 in breast cancer cells. EMBO J 31: 1985-1998, 2012.

14. Yu X, Zhang X, Dhakal IB, Beggs M, Kadlubar S and Luo D: Induction of cell proliferation and survival genes by estradiol-repressed microRNAs in breast cancer cells. BMC Cancer 12: 29, 2012.

15. Xia H, Sun S, Wang B, Wang T, Liang C, Li G, Huang C, Qi D and Chu X: MiR-143 inhibits NSCLC cell growth and metastasis by targeting Limk1. Int J Mol Sci 15: 11973-11983, 2014.

16. Li R, Zhang L, Jia L, Duan Y, Li Y, Wang J, Bao L and Sha N: MicroRNA-143 targets Syndecan-1 to repress cell growth in melanoma. PLoS One 9: e94855, 2014.

17. Wu XL, Cheng B, Li PY, Huang HJ, Zhao Q, Dan ZL, Tian DA and Zhang P: MicroRNA-143 suppresses gastric cancer cell growth and induces apoptosis by targeting COX-2. World J Gastroenterol 19: 7758-7765, 2013.

18. Zhang N, Su Y and Xu L: Targeting PKCe by miR-143 regulates cell apoptosis in lung cancer. FEBS Lett 587: 3661-3667, 2013.

19. Zhang Y, Wang Z, Chen M, Peng L, Wang X, Ma Q, Ma F and Jiang B: MicroRNA-143 targets MACC1 to inhibit cell invasion and migration in colorectal cancer. Mol Cancer 11: 23, 2012.

20. Xu B, Niu X, Zhang X, Tao J, Wu D, Wang Z, Li P, Zhang W, Wu H, Feng N, et al: MiR-143 decreases prostate cancer cells proliferation and migration and enhances their sensitivity to docetaxel through suppression of KRAS. Mol Cell Biochem 350: 207-213, 2011. 